Document downloaded from:

http://hdl.handle.net/10251/143880

This paper must be cited as:

Li, M.; Yan, Z.; Zhu, R.; Phillips, DL.; Aparici-Espert, Ml.; Lhiaubet, VL.; Miranda Alonso, MA. (02-0). Enhanced Drug Photosafety by Interchromophoric Interaction Due to Intramolecular Charge Separation. Chemistry - A European Journal. 24(25):6654-6659. https://doi.org/10.1002/chem.201800716

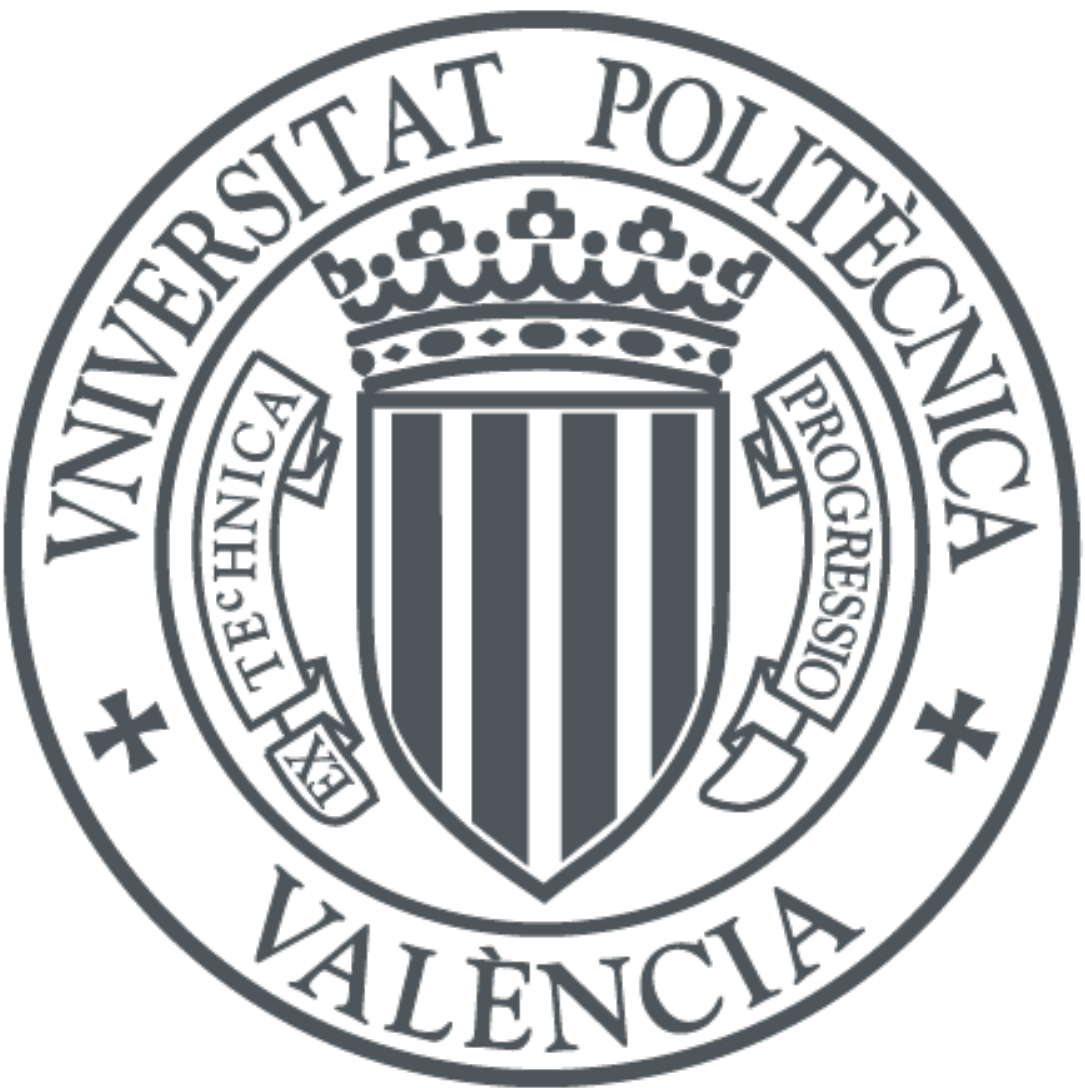

The final publication is available at

https://doi.org/10.1002/chem.201800716

Copyright John Wiley \& Sons

Additional Information 


\title{
Enhanced Drug Photosafety by Interchromophoric Interaction Owing to Intramolecular Charge Separation
}

\author{
Ming-De Li, ${ }^{*[a]}$ Zhiping Yan, ${ }^{[b]}$ Ruixue Zhu, ${ }^{[b]}$ David Lee Phillips, ${ }^{*[a, ~ b] ~}$ Isabel Aparici-Espert, ${ }^{[c]}$ \\ Virginie Lhiaubet-Vallet, ${ }^{*[c]}$ and Miguel A. Miranda*[c]
}

\begin{abstract}
Imatinib is a synthetic tyrosinase inhibitor that is employed for the treatment of some kinds of human cancer. This drug has a low phototoxicity towards DNA, but its pyridylpyrimidine (1) fragment by itself exhibits significant phototoxicitiy. The intrinsic mechanism that leads to the enhanced photosafety of Imatinib is not yet known. Here, the properties of the excited state and interchromophoric interactions of Imatinib have been explored by using ultrafast laser flash photolysis and agarose electrophoresis studies. An intramolecular charge separation was directly observed
\end{abstract}

\section{Introduction}

Imatinib (IMT, Scheme 1) is an efficient tyrosine kinase inhibitor that is widely prescribed for the treatment of Philadelphia chromosome-positive chronic myeloid leukemia or gastrointestinal stromal tumors. ${ }^{[1-5]}$ Interestingly, this drug, labeled as a "magic bullet", is the result of rational design to optimize its chemical structure on the basis of protein kinase inhibition. ${ }^{[3-8]}$ However, cutaneous reactions have been reported by patients who were being treated long term; these included increased photosensitivity, ${ }^{[9-11]}$ cutaneous reactions, ${ }^{[11]}$ psoriasis, ${ }^{[12]}$ pseudoporphyria, ${ }^{[13-15]}$ and squamous cell carcinoma. ${ }^{[16]}$ The reason why Imatinib caused such cutaneous photosensitivity is not clear. Being a photosensitizing drug, its intrinsic photoreactivity and excited-state interactions with biomolecules are of para-

[a] Prof. M.-D. Li, Prof. D. L. Phillips

Department of Chemistry and Key Laboratory for Preparation and Application of Ordered Structural Materials of Guangdong Province Shantou University, Guangdong 515063 (P. R. China) E-mail: mdli@stu.edu.cn

[b] Z. Yan, Dr. R. Zhu, Prof. D. L. Phillips

Department of Chemistry, The University of Hong Kong

Pokfulam Road, Hong Kong S. A. R. (P. R. China) E-mail: phillips@hku.hk

[c] Dr. I. Aparici-Espert, Prof. V. Lhiaubet-Vallet, Prof. M. A. Miranda Instituto de Tecnologia Qu\&mica UPV-CSIC

Universitat PolitHcnica de ValHncia

Consejo Superior de Investigaciones Cientificas

Avda de los Naranjos s/n, 46022 Valencia (Spain)

E-mail: Ivirgini@itq.upv.es

mmiranda@qim.upv.es for the irradiated Imatinib, which accounts for the relaxation of its excited state. An anionic form of pyridylpyrimidine (1) was deduced from the results of time-resolved resonance Raman spectra and by quenching experimental studies on compound 1 and diaminotoluene. In contrast, compound 1 efficiently transformed into triplet excited states with a long lifetime, which explained the phototoxicity associated with this fragment. This work provides insight into how to design drugs with lower phototoxicitiy or improved photostability by using interchromophoric interactions.
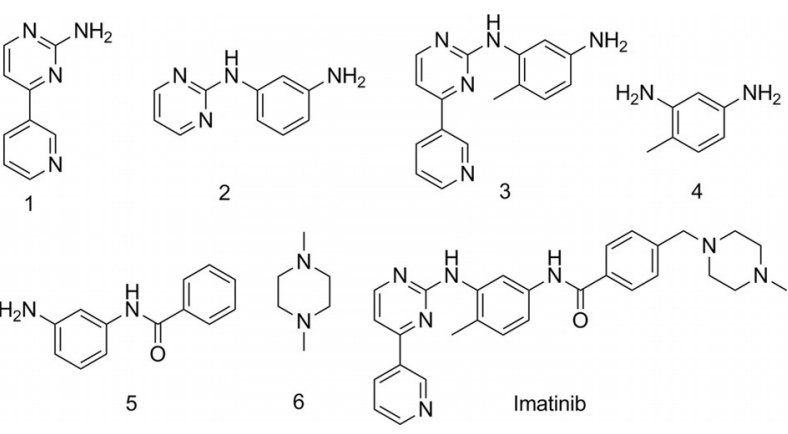

Scheme 1. Imatinib (IMT) and its model substructure molecules 1-6.

mount importance. In this context, a photophysical and photobiological study of IMT and its key chromophores was performed recently, and the results revealed that the IMT drug itself and its anilinopyrimidine fragment (2, Scheme 1) were not DNA photosensitizers. ${ }^{[17]}$ In contrast, the pyridylpyrimidine substructure (1) displayed a marked phototoxic potential that appeared to be associated with the generation of a long-lived triplet excited state. ${ }^{[17]}$ Interestingly, this reactive species was efficiently quenched by 3 -aminobenzanilide (5), which is another molecular fragment of IMT. ${ }^{[17]}$ Integration of the photoactive pyridylpyrimidine moiety into a more complex structure strongly modifies its photobehavior and leads to an improved toxicological profile of IMT. However, it is not clear how the intramolecular interaction between the different chromophores of IMT occur. Interchromophoric interaction is an omnipotent process that is responsible for the electron, proton, energy, and charge transfers among organic light-emitting diodes (OLED), supramolecular frameworks, metal-organic frameworks, light-harvesting systems, and biomolecules. ${ }^{[18-22]}$ Never- 
theless, there are few examples that unveil the nature of an interchromophoric interaction. In an attempt to understand the unexplained photobehavior of IMT and the chromophorechromophore interactions among its substructure molecules (Scheme 1), an investigation has been conducted herein by using femto-to-nanosecond transient absorption, picosecond time-resolved resonance Raman spectroscopy ( $\left.\mathrm{ps}-\mathrm{TR}^{3}\right)$, and nanosecond transient resonance Raman spectroscopy. To better understand the photosafety of IMT, DNA was used as a model substrate to explore the photochemical reactions between IMT and DNA. This study may promote the advancement of the drug discovery and development process. ${ }^{[23]}$ Similar ultrafast spectroscopy measurements have been used to directly observe the photophysical and photochemical processes from femtoseconds to final products for very fast reac- tions. $^{\text {[24- }}$ 26]

\section{Results and Discussion}

Femtosecond transient absorption (fs-TA) spectra were performed to follow the evolution of the excited states of IMT in a PBS (phosphate-buffered saline) solution by using $267 \mathrm{~nm}$ photoexcitation (Figure 1). There are three distinct processes after photoexcitation: Firstly, a transient feature at $362 \mathrm{~nm}$ increased quickly from 0.18 to 0.83 ps (Figure 1 a); this transient was associated with the first singlet excited state of IMT. Figure S1 (see the Supporting Information) shows the calculated electronic spectrum of the first excited states of IMT from TDB3LPY/6-311G (d,p) computations, which matches very well with the transient absorption obtained at early delay times. Secondly, from 0.97 to 1.93 ps, the strong transient absorption intensity at $362 \mathrm{~nm}$ dropped a little bit, and a broad transient absorption maximum at $550 \mathrm{~nm}$ developed and shifted down to $542 \mathrm{~nm}$. This second process was assigned to an intramolecular charge separation between the benzanilide and pyridyl-
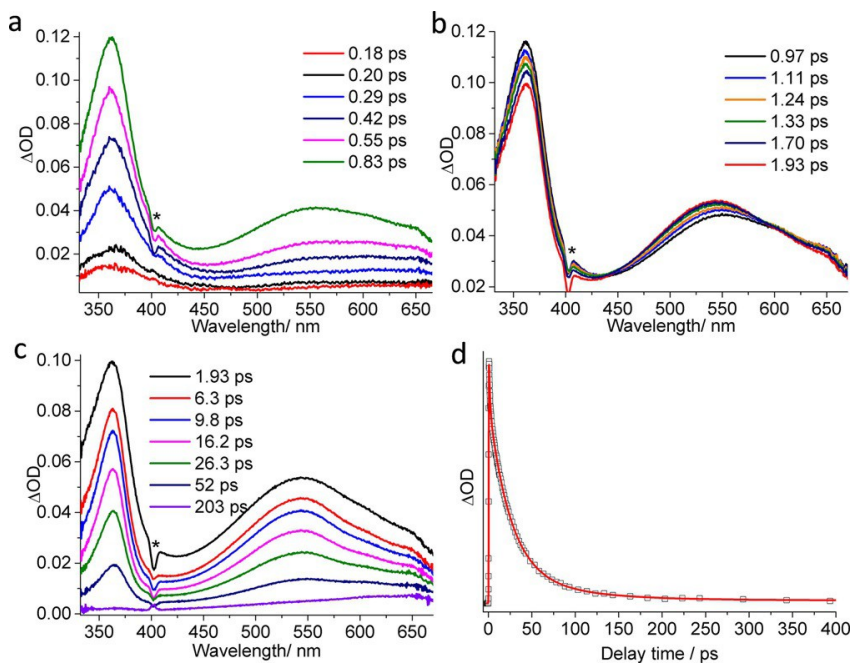

Figure 1. Fs-TA spectra of species obtained in a MeCN/PBS solution acquired after irradiation of IMT at $267 \mathrm{~nm}$ : a) 0.18 to $0.83 \mathrm{ps}$; b) 0.97 to $1.93 \mathrm{ps;}$

c) 1.93 to $203 \mathrm{ps}$; d) kinetics of the characteristic fs-TA absorption band observed at $362 \mathrm{~nm}$. Asterisks represent scattered-light artifacts in the experiment. pyrimidine moieties (see below). Thirdly, the charge recombination led to the consumption of the transient features (362 and $542 \mathrm{~nm}$ ). Both the first singlet excited state and chargeseparation state contributed to the transient absorptions bands at 362 and $542 \mathrm{~nm}$. Three time constants with the amplitudes of 1.1 (24\%), 24 (63\%), and 77 ps (12\%) for $361 \mathrm{~nm}$ were fitted by using a tri-exponential model. After the formation of the excited state and initial relaxation on the subpicosecond timescale, the decay of the excited state and the formation of the charge transfer state occurred within $1.1 \mathrm{ps}$. The decay of the charge transfer state should be related to the longer time constants. Given that the experiment was performed under magic angle, polarization signals that are due to orientational redistribution would be absent. The two time constants, 24 and 77 ps, could be associated with the charge recombination processes that are due to a structural heterogeneity of the IMT molecule. Within 200 ps, the transient features at 362 and $542 \mathrm{~nm}$ had completely disappeared.

The photophysical and photochemical pathways of IMT were also investigated by using $\mathrm{ps}^{-\mathrm{TR}^{3}}$ experiments to explore the nature of its excited states. Figure $2 \mathrm{a}$ shows the ps-TR spectra that was obtained in a PBS solution by using 267 and $365 \mathrm{~nm}$ as the pump and probe wavelengths, respectively. Two main transient Raman bands were observed from 0 to 100 ps. Figure $2 \mathrm{~b}$ shows the comparison between the transient resonance Raman spectra recorded at 0 and 8 ps for IMT alongside the resonance Raman spectra of the model substructure molecules 1 and 2, and IMT. It revealed that the $\mathrm{C}=\mathrm{C}$ stretching vibration Raman band shifted from about $1588 \mathrm{~cm}^{\circledR 1}$ in the ground states of compounds 1, 2, and IMT down to $1542 \mathrm{~cm}^{\circledR 1}$ in the IMT excited states, which indicates that the electronic structure of the drug excited states are significantly different from that of its ground state. A charge separation was pro-
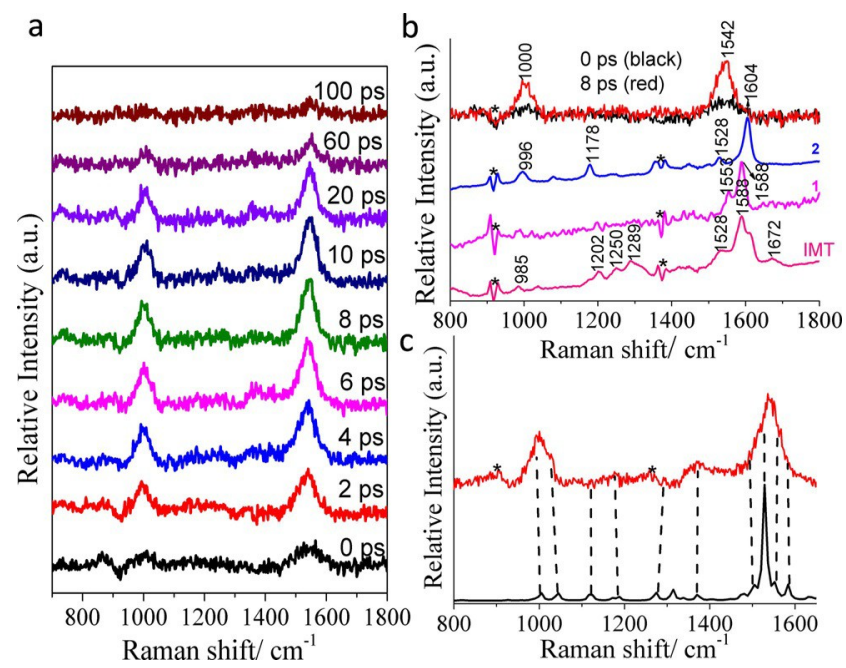

Figure 2. a) Ps-TR 3 spectra obtained at various delay times after photoexcitation of IMT at $267 \mathrm{~nm}$ by using $365 \mathrm{~nm}$ as the probe wavelength in a MeCN/ PBS solution (1:1). b) Comparison of RR spectra recorded at 0 and $8 \mathrm{ps}$ after excitation at $267 \mathrm{~nm}$, and RR spectra of model substructure molecules 1, 2, and IMT in their ground state. c) Comparison of the RR spectrum obtained at $10 \mathrm{ps}$ in a MeCN/PBS solution (1:1; top), and the calculated normal Raman spectrum of the anionic form of the model substructure molecule 1 (bottom). Asterisks represent solvent-subtraction artifacts in the experiment. 
posed to take place from the benzanilide to the pyridylpyrimidine moieties. Figure $2 \mathrm{c}$ shows the comparison of the transient resonance Raman band at $10 \mathrm{ps}$ and the calculated normal Raman band of the anion form of the model substructure molecule 1 . The $C=C$ stretching vibrational mode at $1588 \mathrm{~cm}^{\circledR 1}$ was not enhanced, whereas the resonance at $1542 \mathrm{~cm}^{\circledR 1}$ was selectively enhanced when the charge separation occurred. This demonstrates that the charge separation plays a significant role in the electronic structural change of the anion form of model molecule 1 . Figure 3 shows a comparison of the opti-

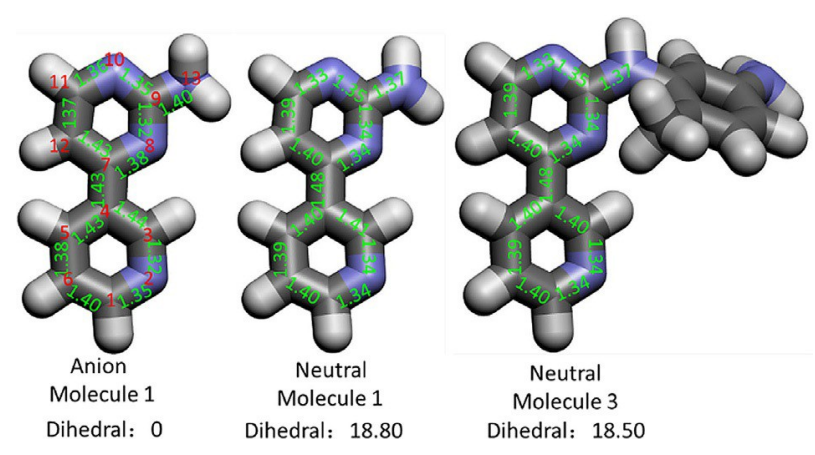

Figure 3. Optimized structures of the anionic form of the substructure molecule 1 (left) and the neutral form of substructure molecules 1 and 3 . The bond lengths and the dihedral angles between the planes of the two phenyl rings are listed.

mized structures of anion 1 and neutral systems 1 and 3. Careful examination of these structures revealed that the dihedral angle between the two phenyl rings of anion 1 is 08 , whereas that between the two phenyl rings of the neutral molecules 1 and 3 is about 188. Furthermore, most of the bond lengths changed significantly owing to the electron becoming delocalized into the phenyl ring when the charge separation takes place; this delocalization makes these rings more conjugated, and the $\mathrm{p}$ system will consequently twist the two phenyl rings into the same plane.

To better understand the proposed charge-separation process of IMT, the nature of the excited states for the substructure molecules 1 and 3 was investigated with fs-TA. Figure 4 displays the fs-TA spectra of substructure molecule 1; after photoexcitation, it exhibited different spectra profiles from those of the parent drug. Initially, it was promoted to the first excited state, which resulted in a strong transient absorption at $358 \mathrm{~nm}$ and a weak broad tail band around $510 \mathrm{~nm}$ (Figure 4 a, 1.1 ps spectrum); then, it underwent a vibrational cooling process from 1.1 to 73.6 ps. At later times, the first singlet excited state underwent an intersystem crossing to transform into the triplet excited state with transient absorption bands at 330 and $444 \mathrm{~nm}$, which is consistent with the nanosecond transient absorption features that were previously reported. ${ }^{[16]}$ Fitting of the tri-exponential function of the time-dependent kinetics at 362 and $445 \mathrm{~nm}$ established the existence of three time constants: 0.54 (7\%), 61 (11\%), and 1270 ps (66 \%), which are associated with the promotion to the first excited state, vibrational cooling, and intersystem crossing, respectively.
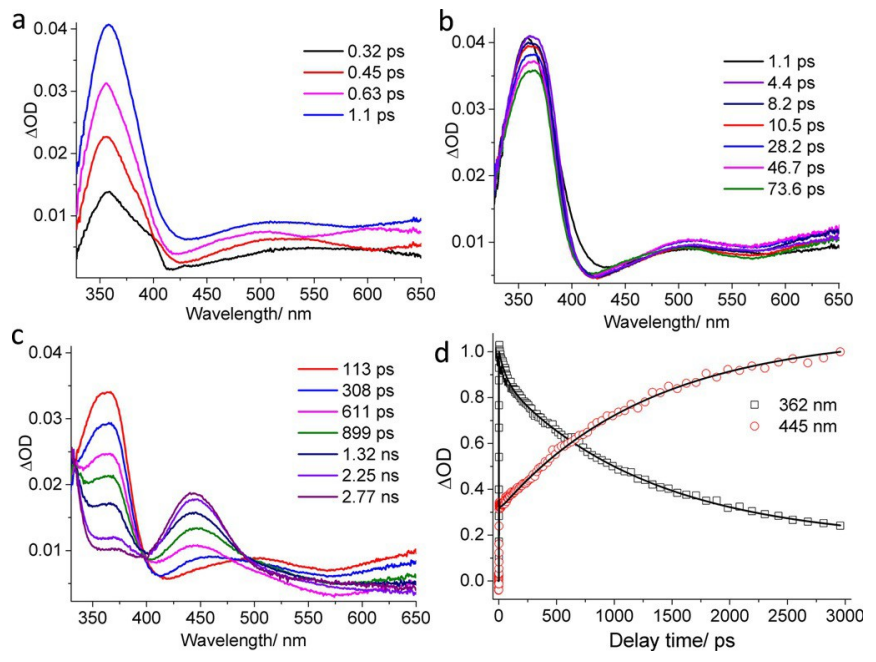

Figure 4. Fs-TA spectra of molecule 1 at $267 \mathrm{~nm}$ in a MeCN/PBS solution: a) 0.32 to $1.1 \mathrm{ps}$; b) 1.1 to $73.6 \mathrm{ps}$; c) $113 \mathrm{ps}$ to $2.77 \mathrm{~ns}$. d) Kinetics of the characteristic fs-TA absorption bands observed at $362 \mathrm{~nm}$ and $445 \mathrm{~nm}$ after photoexcitation of molecule 1 at $267 \mathrm{~nm}$ in a MeCN/PBS solution.

To confirm the triplet excited state assignment for substructure molecule 1, we also calculated the Raman spectrum of its triplet state. Figure S2 (see the Supporting Information) displays the comparison of the calculated Raman spectrum and the high-low power transient resonance Raman spectrum by using $266 \mathrm{~nm}$ as the excitation wavelength. The reasonable agreement of those two spectra further supports that the species detected after several nanoseconds is a triplet excited state. However, when substructure molecules 1 and 2 were fused, as found in compound 3 and IMT, the intersystem-crossing channel was efficiently shut down and the charge-separation channel became the predominant process. Figure S3 (see the Supporting Information) shows the fs-TA spectra of substructure molecule 3; from 0.27 to $0.93 \mathrm{ps,}$, population of the first singlet excited state resulted in a main transient absorption around $365 \mathrm{~nm}$; then, it undergoes charge separation, like the behavior observed for IMT in Figure 1. Figure S3 b (see the Supporting Information) shows that the intensity of the transient band at $365 \mathrm{~nm}$ dropped a little bit and a broad shoulder feature at $542 \mathrm{~nm}$ remained constant. Finally, the 365 and $542 \mathrm{~nm}$ transient bands completely disappeared within 18.5 ps. This process was brought about by the charge recombination. Two time constants, 0.20 and 2.4 ps, were simulated by the two-exponential fitting of transient absorptions at $365 \mathrm{~nm} .0 .20 \mathrm{ps}$ lifetime was attributed to the growth of first singlet excited state and charge separation, whereas 2.4 ps time constant was associated with the charge recombination. The features of the fs-TA spectra of the substructure molecule 3 were very similar to those of IMT in the acetonitrile/PBS solution, except that the decay kinetics for compound 3 were faster than that of IMT. This indicates that the charge-separation state in compound 3 is not very stable and leads to a faster charge recombination rate than for the larger conjugated system of IMT. The charge-separation state appeared more stable in IMT, which is a larger system that can give rise to a charge transfer towards a side terminal that is at a greater dis- 
tance. So, the charge recombination rate of IMT was slower than that observed for substructure molecule 3 . On the basis of the fs-TA results for IMT and its substructures, the chargeseparation process from benzanilide to the pyridylpyrimidine moieties can mainly account for the low phototoxicity of IMT, whereas the substructure molecule 1 can easily be transformed into a long-lived triplet state after the photoexcitation.

To confirm the charge-separation nature of IMT, nanosecond laser flash photolysis was performed on substructure molecule 4 (diaminotoluene), which is a model for the electron donor moiety of substructure molecule 3 , by using $266 \mathrm{~nm}$ as the excitation wavelength (see the Supporting Information, Figure S4). The species with the broad transient absorption around $700-720 \mathrm{~nm}$ was efficiently quenched by $\mathrm{N}_{2} \mathrm{O}$, as expected for a solvated electron. Thus, under the laser excitation of compound 4, photoionization takes place and results in the formation of a diaminotoluene radical cation, which exhibited a transient absorption centered at $480 \mathrm{~nm}$. This indicates that the substructure molecule 4 is easily oxidized. In contrast, the photoreactivity of fragment 1 was also examined in the presence of good electron donors, such as DABCO, to probe the reactivity of the triplet state of compound 1 . Interestingly, the structure of this amine was similar to that of the dialkyl piperazine moiety (6) that is present in IMT. The results from these laser flash photolysis studies by using $266 \mathrm{~nm}$ as the excitation wavelength showed that compound 1 was easily reduced by $D A B C O$, which gave rise to the radical anion with a redshifted transient absorption spectrum (Figure 5). The spectral signature observed for the pyridylpyrimidine radical anion had central transient absorptions at about 340 and $480 \mathrm{~nm}$. These signals were blueshifted relative to those of the IMT charge-separation state, which displayed central transient absorptions at about 362 and $542 \mathrm{~nm}$; this must be attributed to the different nature of the connecting nitrogen atom (amine vs. aniline).
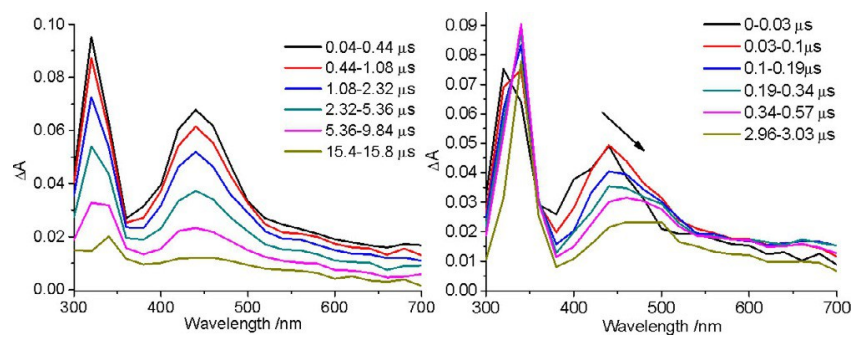

Figure 5. Transient absorption spectra of molecule $1(0.113 \mathrm{~mm})$ in MeCN/ $\mathrm{H}_{2} \mathrm{O}(4: 1, v / v)$ under $\mathrm{N}_{2}$ (left) and in the presence of DABCO $(5.7 \mathrm{~mm})$ under $\mathrm{N}_{2}$ (right).

A parallel experiment was also performed by using $266 \mathrm{~nm}$ excitation of a $\mathrm{N}_{2}$-purged water solution of compound 4 $(0.4 \mathrm{~mm})$ in the presence of an increasing amount of compound 1 (up to $0.085 \mathrm{~mm}$, dissolved in $\mathrm{MeCN} / \mathrm{H}_{2} \mathrm{O}, 4: 1, \mathrm{v} / \mathrm{v}$ ) (see the Supporting Information, Figure S5). No solvated electron was observed under these conditions, and instead, a weak band that peaked at $480 \mathrm{~nm}$ (attributed to $\left.1^{(}\right)$was again observed. Therefore, the diaminotoluene-like moiety of IMT can act as an electron donor, the acceptor being the substructure
1. Moreover, quenching of compound 1 by DABCO also suggests a potential electron transfer between the dialkyl piperazine group (6) and the pyridylpyrimidine moiety in IMT.

Finally, agarose electrophoresis experiments were performed to establish if this charge-separation process between IMT substructures may be responsible for its remarkably enhanced drug photosafety. Thus, DNA ( $9 \mathrm{~nm}, 38 \mathrm{~mm}$ in base pair) was UVA irradiated in the presence of IMT and fragments 1 and 3 $(80 \mathrm{~mm})$. As shown in Figure 6, single-strand breaks were only observed in the case of the pyridylpyrimidine derivative 1 , whereas no photogenotoxicity was observed for the experiment with IMT and compound 3, which exhibited the same band pattern as DNA alone. These results corroborate the deactivation pathway that is triggered by the charge-separation process.

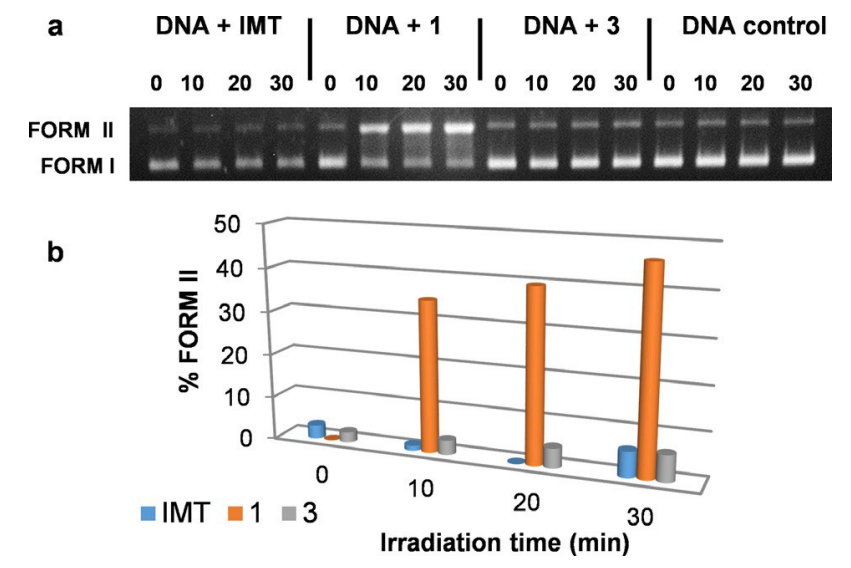

Figure 6. a) Agarose gel electrophoresis of UVA-irradiated samples of plasmid DNA ( $9 \mathrm{~nm}, 38 \mathrm{~mm}$ in base pair) alone or in the presence of IMT, 1, or 3 $(80 \mathrm{~mm})$. b) Quantification of the $\%$ of form II as a function of irradiation time.

\section{Conclusion}

Exploration of the excited states and transient states of IMT and substructures 1 and 3 by using transient absorption and time-resolved resonance Raman spectroscopy revealed that an intramolecular charge separation takes place from the benzanilide to the pyridylpyrimidine moieties upon the illuminated IMT, which accounts for the relaxation of its excited state. An anionic form of pyridylpyrimidine was directly observed by time-resolved resonance Raman spectra and quenching experimental studies on IMT, pyridylpyrimidine, and diaminotoluene. However, the singlet excited states efficiently transformed into triplet excited states with a long lifetime upon the photoexcitation of pyridylpyrimidine. Long lifetime triplet excited states were responsible for the phototoxicity associated with pyridylpyrimidine. Agarose electrophoresis studies on IMT and substructures 1 and 3 found that, upon UVA irradiation, the singlestrand breaks were only formed in the case of the pyridylpyrimidine derivative, whereas no photogenotoxicity was observed for IMT and substructure 3. This study paves the way for the development of principles for the design of drugs and related molecules that have lower phototoxicitiy or improved 
photostability by using chromophore-chromophore interactions.

\section{Experimental Section}

\section{Materials}

The IMT was purchased from KEMPROTEC Limited. Fragment 1 was purchased from Fluorochem. Compounds 2 and 4 were purchased from Sigma-Aldrich and fragment 3 was purchased from ABCR or Santa Cruz Biotechnology. Spectroscopic-grade acetonitrile (MeCN) and deionized water were used to prepare the sample solutions for use in the time-resolved spectroscopy experiments.

\section{Methods}

Femtosecond transient absorption spectroscopy (fs-TA): Experiments were performed by employing an experimental setup and methods detailed previously; ${ }^{[27]}$ only a brief description is provided here. Fs-TA measurements were done by using a femtosecond regenerative amplified Ti:sapphire laser system, in which the amplifier was seeded with the $120 \mathrm{fs}$ laser pulses from an oscillator laser system. The laser probe pulse was produced by utilizing $5 \%$ of the amplified $800 \mathrm{~nm}$ laser pulses to generate a white-light continuum (330-800 nm) in a $\mathrm{CaF}_{2}$ crystal; this probe beam was split into two parts before traversing the sample. One probe laser beam goes through the sample, and the other beam goes to the reference spectrometer to monitor the fluctuations in the probe beam intensity. For the experiments discussed in this work, a $40 \mathrm{~mL}$ solution was flowed through a cuvette with a $2 \mathrm{~mm}$ path length. This flowing sample was then excited by a $267 \mathrm{~nm}$ pump laser beam. An absorbance of compound 1 at $267 \mathrm{~nm}$ was used for the sample solutions in fs-TA experiments to maintain the same number of photons being absorbed and the same irradiating conditions for each sample.

Picosecond time-resolved resonance Raman spectroscopy (ps$T^{3}$ ): Experiments were done by employing picosecond regenerative amplified Ti:sapphire laser system. The laser probe pulse was produced by the picosecond model TOPAS by utilizing $80 \%$ of the amplified $800 \mathrm{~nm}$ laser that was pulsed to generate $365 \mathrm{~nm}$ with $25 \mathrm{~mW}$; this probe beam was used to selectively enhance the resonance Raman signal of excited states and transient intermediates. The laser pump pulse was produced by the third harmonic of $20 \%$ amplified $800 \mathrm{~nm}$ laser that was pulsed to generate $267 \mathrm{~nm}$ to excite the sample. The pump pulse photoexcited the sample to initiate the photochemical processes, and the probe pulse monitored the sample and the intermediate species formed. The laser beams were lightly focused and lined up so that they merged together onto a flowing sample. A traveling delay stage was put in the pump beam path to set the time delay between the pump and probe laser pulses. The Raman scattered signal was collected by using a backscattering geometry and observed by a liquid-nitrogen-cooled charge-coupled device (CCD) detector. The ps-TR ${ }^{3}$ spectra shown here were derived from the subtraction of an appropriately scaled probe-before-pump spectrum from the correlated pump-probe resonance Raman spectrum to mostly remove nontransient bands. The Raman bands of $\mathrm{MeCN}$ were used to calibrate the Raman shifts with an estimated uncertainty of $5 \mathrm{~cm}^{\circledR 1}$. The sample concentrations in $\mathrm{ps}-\mathrm{TR}^{3}$ were \& $5 \mathrm{~V} 10^{\circledR 4} \mathrm{~m}$.

Nanosecond transient resonance Raman and resonance Raman spectroscopy (RR): Experiments were done by employing the fourth harmonic of a Nd:YAG nanosecond pulsed laser, which supplied the $266 \mathrm{~nm}$ wavelength. The laser beams were lightly fo- cused onto a flowing sample. The Raman scattered signal was collected by using a backscattering geometry and observed by a liquid-nitrogen-cooled charge-coupled device (CCD) detector. Transient resonance Raman experiments were performed by using high and low power of $266 \mathrm{~nm}$ excitation wavelength. Transient resonance Raman signal was obtained by the subtraction of the resonance Raman spectrum of the sample in low-power mode from the resonance Raman spectrum of the sample in high-power mode, by which the signal of the ground state of the sample was eliminated completely. The RR spectra shown here were found from the subtraction of an appropriately scaled spectrum of just the solvent from the correlated resonance Raman spectrum of the solvent with the sample to mostly remove solvent bands. The Raman bands of MeCN were used to calibrate the Raman shifts with an estimated uncertainty of $5 \mathrm{~cm}^{\circledR 1}$. The sample concentrations in RR were \& $5 \mathrm{~V} 10^{\circledR 4} \mathrm{M}$.

Laser flash photolysis (LFP): Experiments were performed with a pulsed Nd:YAG (L52137 V LOTIS TII) laser system instrument by setting $266 \mathrm{~nm}$ as the excitation wavelength. The pulse duration was of about $10 \mathrm{~ns}$ and the energy was adjusted at $29 \mathrm{mJpuls} \mathrm{e}^{\circledR 1}$. The apparatus was composed of the pulsed laser, the Xe lamp, a 77250 Oriel monochromator, and a photomultiplier. The output signal from a Tektronix oscilloscope was transferred to a personal computer. The transient spectra were recorded at RT by employing quartz cells with an optical path length of $1 \mathrm{~cm}$. In the case of diaminotoluene $4(0.4 \mathrm{~mm})$, experiments were performed in $\mathrm{H}_{2} \mathrm{O}$ under aerobic and anaerobic conditions by using $\mathrm{N}_{2}$ or $\mathrm{N}_{2} \mathrm{O}$. Transient absorption spectra of compound $4(0.4 \mathrm{~mm})$ were also registered in the presence of fragment 1 (up to $0.085 \mathrm{~mm}$ dissolved in $\mathrm{MeCN} / \mathrm{H}_{2} \mathrm{O}, 4: 1, \mathrm{v} / \mathrm{v}$ ) in $\mathrm{N}_{2}$-purged water. Experiments carried out on the fragment $1(0.113 \mathrm{~mm})$ were performed in $\mathrm{MeCN} / \mathrm{H}_{2} \mathrm{O}, 4: 1$. Quenching experiments of the transient species of fragment 1 were performed by adding DABCO (from 0 to $5.7 \mathrm{~mm}$ ), and the bimolecular rate constant $k_{\mathrm{q}}$, which was determined by using the SternVolmer equation, was calculated to be about $8 \vee 10^{8} \mathrm{~m}^{\circledR 1} \mathrm{~s}^{\circledR 1}$ for DABCO.

\section{Plasmid DNA damage/Agarose gel electrophoresis}

Samples that contained supercoiled circular DNA (pBR322, 4361 base pair; $5 \mathrm{~mL}, 9 \mathrm{~nm}, 38 \mathrm{~mm}$ in base pair) of in the absence and presence of IMT, 1 , or $3(80 \mathrm{~mm})$ were employed in electrophoresis experiments. The samples were irradiated by using a multilamp photoreactor with lamps that emitted in the $300-400 \mathrm{~nm}$ range with a maximum at $355 \mathrm{~nm}$. The samples were loaded on a $1 \%$ agarose gel that contained SYBR Safe. After electrophoresis, the relative abundance of supercoiled DNA (form I) and relaxed DNA (form II) was quantified by densitometry. The yield of a singlestrand break after $30 \mathrm{~min}$ of irradiation in the presence of fragment 1 was $46 \%$, whereas it was of about $6 \%$ for IMT and fragment 3 .

\section{Computational studies}

The TD-DFT methodology (TD-B3LYP/6-311G(d,p)) in PCM solvent mode was performed to predict the UV/Vis absorption spectra of the candidate transient species that was generated from the photolysis of the IMT. GaussSum software was utilized to simulate the UV/Vis spectra. ${ }^{[28]}$ To predict the $\mathrm{TR}^{3}$ spectra, second-order MøllerPlesset perturbation theory (MP2) with a $6-311 G(d, p)$ basis set was employed to optimize the structures and predict the Raman spectra of the anionic form of fragment 1. A Lorentzian function with a $15 \mathrm{~cm}^{\circledR 1}$ bandwidth for the vibrational frequencies and a frequency scaling factor of 0.974 was used in the comparison of the calculated results with the experimental spectra. ${ }^{[29]}$ The other structures 
(neutral forms of fragments 1 and 3) were optimized by the DFT (B3LYP/6-311G(d,p)) methodology. No imaginary frequency modes were observed at the stationary states of the optimized structures. All of the calculations were done by using the Gaussian 09 program. ${ }^{[30]}$

\section{Acknowledgements}

This work was supported by Natural Science Foundation of China (21773151) and Shantou University Initial Funding (NTF16010). The Support from the Hong Kong Research Grants Council grants GRF 17307916, AoE/P-03/08, SEG HKU/07, The University of Hong Kong Development Fund 2013-2014 project "New Ultrafast Spectroscopy Experiments for Shared Facilities", the Spanish Government (CTQ2015-70164-P and BES- 2013066566) are also acknowledged.

\section{Conflict of interest}

The authors declare no conflict of interest.

[1] F. Stegmeier, M. Warmuth, W. R. Sellers, M. Dorsch, Clin. Pharmacol. Ther 2010, 87, $543-552$.

[2] S. Patel, Cancer Chemother. Pharmacol. 2013, 72, 277 -286.

[3] N. Lydon, Nat. Med. 2009, 15, 1153 - 1157.

[4] M. Bucci, C. Goodman, T. L. Sheppard, Nat. Chem. Biol. 2010, 6, $847-$ 854.

[5] J. C. Yang, D. Niu, B. P. Karsten, F. Lima, S. L. Buchwald, Angew. Chem. Int. Ed. 2016, 55, 2531-2535; Angew. Chem. 2016, 128, 2577-2581.

[6] S. Ömura, Angew. Chem. Int. Ed. 2016, 55, 10190-10209; Angew. Chem. 2016, 128, 10344- 10364.

[7] K. M. Korch, C. Eidamshaus, D. C. Behenna, S. Nam, D. Horne, B. M Stoltz, Angew. Chem. Int. Ed. 2015, 54, 179 -183; Angew. Chem. 2015, 127, $181-185$

[8] N. B. Lydon, B. J. Druker, Leukemia Res. 2004, 28S1, S29 -S38.

[9] I. Amitay-Laish, S. M. Stemmer, M. E. Lacouture, Dermatol. Ther. 2011, 24, $386-395$.

[10] P. Rousselot, J. Larghero, E. Raffoux, F. Calvo, M. Tulliez, S. Giraudier, M. Rybojad, Br. J. Haematol. 2003, 120, 1091 -1092.

[11] V. Brazzelli, F. Muzio, G. Manna, E. Moggio, C. Vassallo, E. Orlandi, G. Fiandrino, M. Lucioni, G. Borroni, Photodermatol. Photoimmunol. Photomed. 2012, 28, 2-5.

[12] F. Atalay, E. KızılkılıÅ, R. S. Ada, Turk. J. Haematol. 2013, 30, 216 -218.
[13] L. Valeyrie, S. Bastuji-Garin, J. Revuz, N. Bachot, J. Wechsler, P. Berthaud, M. Tulliez, S. Giraudier, J. Am. Acad. Dermatol. 2003, 48, $201-206$.

[14] N. Heidary, H. Naik, S. Burgin, J. Am. Acad. Dermatol. 2008, 58, 545-570.

[15] L. Timmer-de Mik, S. H. Kardaun, M. H. H. Kramer, D. P. Hayes, M. T. Bousema, Clin. Exp. Dermatol. 2009, 34, 705 -707.

[16] G. Baskaynak, K. A. Kreuzer, M. Schwarz, J. Zuber, H. Audring, H. Riess, B. Dorken, P. Le Coutre, Eur. J. Haematol. 2003, 70, 231 -234.

[17] G. Nardi, V. Lhiaubet-Vallet, M. A. Miranda, Chem. Res. Toxicol. 2014, 27, 1990-1995.

[18] P.Deria, J. Yu, T. Smith, R. P. Balaraman, J. Am. Chem. Soc. 2017, 139, 5973-5983.

[19] Y. Jiang, J. McNeill, Chem. Rev. 2017, 117, 838-859.

[20] U. Lewandowska, W. Zajaczkowski, L. Chen, F. BouilliHre, D. Wang, K. Koynov, W. Pisula, K. Mellen, H. Wennemers, Angew. Chem. Int. Ed. 2014, 53, 12537-12541; Angew. Chem. 2014, 126, 12745-12749.

[21] B. Liu, W. Zhao, C. Y. Luo, F. Lu, S. L. Tao, Q. X. Tong, J. Mater. Chem. C 2016, 4, $2003-2010$

[22] C. Fang, R. Frontiera, R. Tran, R. A. Mathies, Nature 2009, 462, 200-204.

[23] K. C. Nicolaou, Angew. Chem. Int. Ed. 2014, 53, 9128-9140; Angew. Chem. 2014, 126, $9280-9292$.

[24] M. D. Li, P.J. Hanway, T. R. Albright, A. H. Winter, D. L. Phillips, J. Am. Chem. Soc. 2014, 136, $12364-12370$.

[25] M. D. Li, T. R. Albright, P. J. Hanway, M. Liu, X. Lan, S. Li, J. Peterson, A. H. Winter, D. L. Phillips, J. Am. Chem. Soc. 2015, 137, 10391-10398.

[26] L. L. Du, Y. Qiu, X. Lan, R. Zhu, D. L. Phillips, M. D. Li, A. S. Dutton, A. H. Winter, J. Am. Chem. Soc. 2017, 139, 15054-15059.

[27] M. D. Li, J. Ma, T. Su, M. Liu, L. Yu, D. L. Phillips, J. Phys. Chem. B 2012, $116,5882-5887$.

[28] N. M. O'boyle, A. L. Tenderholt, K. M. Langner, J. Comput. Chem. 2008 $29,839-845$.

[29] J. P. Merrick, D. Moran, L. Radom, J. Phys. Chem. A 2007, 111, $11683-$ 11700.

[30] Gaussian 09, Revision A.02, M. J. Frisch, G. W. Trucks, H. B. Schlegel, G. E. Scuseria, M. A. Robb, J. R. Cheeseman, G. Scalmani, V. Barone, B. Mennucci, G. A. Petersson, H. Nakatsuji, M. Caricato, X. Li, H. P. Hratchian, A. F. Izmaylov, J. Bloino, G. Zheng, J. L. Sonnenberg, M. Hada, M. Ehara, K. Toyota, R. Fukuda, J. Hasegawa, M. Ishida, T. Nakajima, Y. Honda, O. Kitao, H. Nakai, T. Vreven, J. A. Montgomery, Jr., J. E. Peralta, F. Ogliaro, M. Bearpark, J. J. Heyd, E. Brothers, K. N. Kudin, V. N. Staroverov, R. Kobayashi, J. Normand, K. Raghavachari, A. Rendell, J. C. Burant, S. S. Iyengar, J. Tomasi, M. Cossi, N. Rega, J. M. Millam, M. Klene, J. E. Knox, J. B. Cross, V. Bakken, C. Adamo, J. Jaramillo, R. Gomperts, R. E. Stratmann, O. Yazyev, A. J. Austin, R. Cammi, C. Pomelli, J. W. Ochterski, R. L. Martin, K. Morokuma, V. G. Zakrzewski, G. A. Voth, P. Salvador, J. J. Dannenberg, S. Dapprich, A. D. Daniels, Z. Farkas, J. B. Foresman, J. V. Ortiz, J. Cioslowski, D. J. Fox, Gaussian, Inc. Wallingford CT, 2009. 